\title{
Grey - mutation Model of Residual Error Correction is Applied to the Landslide time Prediction Research
}

\author{
Fuyou Liu \\ Henan province prospecting bureau of geology and \\ mineral resources line \\ The second survey institute of geology and mineral \\ Zhengzhou, China \\ 895368014@qq.com \\ Yani Guo* \\ China University of geosciences (Wuhan) \\ Institute of electrical and mechanical \\ Wuhan, China \\ 498914552@qq.com \\ * Corresponding Author
}

\author{
Yong Liu \\ China University of geosciences (Wuhan) \\ Institute of electrical and mechanical \\ Wuhan, China \\ 99086669@qq.com
}

Fengbo Liu

China University of geosciences (Wuhan)

Institute of electrical and mechanical

Wuhan, China

237668254@qq.com

\begin{abstract}
Landslide danger of early warning is to avoid a major means of state personnel and economic losses. In this paper, in order to effectively predict Baishuihe landslide danger instability, combine with the specific engineering practice, the first application of grey theory of landslide displacement data processing, get the forecast data, and calculate the data of landslide displacement and landslide prediction residual value of the data, according to the theory of system engineering science and nonlinear science grey cusp mutation theory, the landslide prediction and residual value is used to detect the mutation respectively, earning their forecasts, to amend the residual prediction data for landslide time value of the mutation happened. By comparing with the test data of Baishuihe landslide. By comparing with the test data of Baishuihe landslide, the residual prediction correction forecast results than those not worth to more accurately predict time shows that the model can be more effective to predict landslide displacement mutation time.
\end{abstract}

Keywords-landslide; time prediction ; grey - cusp mutation theory; residual error correction ; model

\section{INTRODUCTION}

For the traditional landslide time prediction, most of them adopt statistical prediction model, such as Saito model. These models have the following limitations: (1) can only describe the continuous changes of the state; (2) it is a group of qualitative, unique, small disturbance can be ignored; (3) the physicalmeaning is not so clear.

Landslide is a kind of non-continuous mutation phenomenon. The stability of landslide will be changed with the change of control variables, and the critical phenomena occur. Mutation theory [1] is a powerful tool to study the behavior. In view of this, a new theory of non-continuous mutation phenomenon - the mutation theory is used, by analyzing the landslide precursory deformation data, a cusp mutation model [2] is established. On this basis, the grey theory and mutation theory [3] can be integrated, using grey - mutation theory landslide displacement prediction method to predict the time of occurrence of mutations. This paper also introduces the prediction residual correction at the same time, by calculating the residual values of landslide displacement data and landslide forecast data, landslide prediction value and residual values are mutation detection, each receive data prediction and the residual prediction data for correction of lands lide mutations occurred at a time value. This method cannot only correctly describe the nonlinear characteristics of the process of the landslide instability, but also can develop new ideas of instability prediction.

\section{CUSP MUTATION MODEL}

Using the least square method, the form of the single variable function is expressed as a power series form. That is:

(1) $\mathrm{x}$ is considered as time, $\mathrm{y}$ is regarded as corresponding to the displacement $, \cdots, \cdots$; is a constant to be determined. Through the certain trend of regular sequences are analyzed, and find general intercepted four items, the required precision is high enough, and intercept to the four items (1) can be represented as:

(2) Type of variable substitution, the standard form into the cusp mutation, shilling

(3) Type, (3) into (2) available:

Among them:

Through the (4) is a variable substitution, turning it into standard form of cusp mutation, to:

Here, only for analysis, the (6) into (4) are:

(7) As shear, meaningless is in mutation analysis on it. $\mathrm{a}, \mathrm{b}$ are: 
(7) For $\mathrm{z}$ is state variables, namely to $\mathrm{a}, \mathrm{b}$ is control variables of the cusp mutation model. In addition to the standard form of cusp mutation, it is important to find out the important parameters of the system state change, while ignoring the secondary variables. By the theory of mutation, the equilibrium surface equation is:

Bifurcation set equation is:

Only in the control variables, the system is unstable, and the system can be changed from one equilibrium state to another.

The bifurcation set is a half cubic parabola (Fig. 1), the point $(0,0)$ is a point. Control variable plane by bifurcation set is divided into two intervals. In the region of the $\mathrm{E}$ (note (11) left for D), then D>0, system state is stable [4]; in area $\mathrm{J}$, then $\mathrm{D}<0$. The system has three equilibrium points, two of which are stable and the other one is unstable. The unstable points are not achieved in theory, but in the actual process of slope evolution, it is possible to achieve, so when $\mathrm{D}<0$, the slope may be unstable. The standard to determine the point of instability is to meet:

In the right branch of the bifurcation set $(b>0)$, then the mathematical structure of the mutant (the number of equilibrium and stability) is being talked about to have mutations in the system, and $\mathrm{z}$ values are not jumping. Across the bifurcation set for a left branch $(b<0)$ in the case, then the corresponding point is unstable, $\mathrm{z}$ value jumps occur. Now determine the corresponding forked branch on the left set of state variables for each point $\mathrm{z}$ values. Under the condition of (11) is established, when a $=0$, equation (10) has a triple zero root; When a $<0$ there are three real root, they are:

State variable occurs across the bifurcation set jump (Fig. 2):

Corresponding to the slope instability before and after the time difference:

The formula can be used to calculate the slope in the critical state $(D=0)$ and fracture of the time difference on, the and in the critical state duration and that slope failure time. In the second case $(D<0,3 a<0)$, system status bifurcation within the set time to failure is determined by the above method should generally be longer than the actual time to failure.

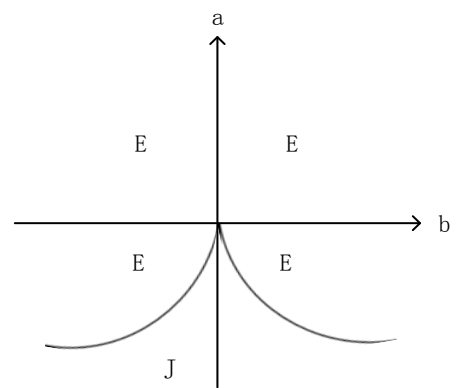

Figure 1. Bifurcation set into control space

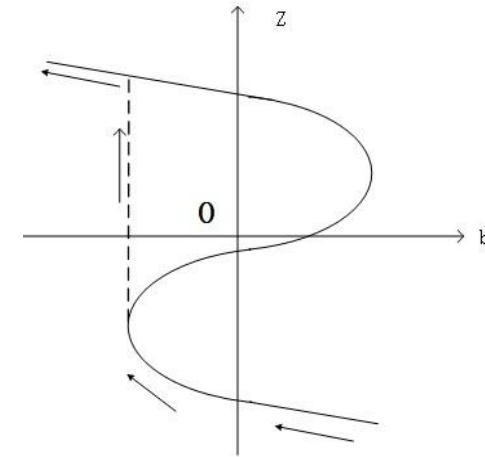

Figure 2. Crossing the bifurcation set of state variables $\mathrm{Z}$ jump

\section{GREY MUTATION THEORY PREDICTION}

The grey system is about information is incomplete or uncertain system control theory, is proposed by Chinese Professor Deng Julong, grey system theory is used to solve the mathematical method of incomplete information system. In view of the present research situation of the gray system theory, the gray model is only suitable for the data sequence is exponential increasing. And the process of the nonlinear problem is relatively weak. Therefore, it is necessary to develop the grey model (single sequence as an example), and enhance its generalization.

Grey modeling theory is the essence of "generation" through the use of information processing method to weaken the original random sequence (grey sequence) of randomness, the transformation of the original random sequence too easy to modeling the new sequence. The generation of grey sequence is not to seek the statistical law of probability, but to strengthen the utilization rate of useful information in grey sequence. In various generating methods, the accumulated generating (AGO) is the basic and commonly used method. So that as a gray time series, for which $\mathrm{AGO}$ sequence:

(18) For k time sequence of grey value, for k AGO time sequence value, can be determined by the type

Set: the power series curve in time $t$ is:

Type on the extended model for gray, the type of parameter is constant to be determined. According to the mean value theorem, grey extended model can within the scope of the requirements of the error instead of grey model. It can solve the problem of grey model can solve, and itself has the property of grey model no. Intercept the (20) to 5 times, can be represented as:

(21) It is approved by a line operation (integral) and the new sequence value resulting from the interception of expression. After reduction of (25) derivative model expression, namely:

(22) The sequence value $y$ is calculated by the reduction model.

To:

Find the original time series and the sequence of values of $y$ after reduction model residuals e (k):

The residuals processed data sequence and the original sequence of data values into (2), and press the second section of the mutation theory and calculation method to determine the stability of slope failure time. Grey theory has its superiority in the data processing, the application of mutation theory analysis of slope evolution has its one's own knack in nature. Integrate the advantages of both. Prediction method using grey mutation theory. This 
method not only broadens the application of mutation theory in slope stability evaluation and time prediction, but also improves the accuracy of prediction.

\section{CASE ANALYSIS}

Baishuihe landslide is in the Three Gorges Reservoir area of the Yangtze River valley wide territory of Zigui County, south to north, the Yangtze River stepped distribution. Landslide is an oversize old loose deposit landslide, development in Zigui syncline west wing, strata occurrence $15^{\circ}, 36^{\circ}$ inclination, for single layer inclined consequent slope (Fig. 3). Landslide rear elevation $410 \mathrm{~m}$, by a geotechnical boundaries; The front elevation of about $70 \mathrm{~m}$, has no storage under water; Things to bedrock ridges is bounded on both sides, about $30^{\circ}$, the overall slope landslide in north and south is about $600 \mathrm{~m}$ long, east-west width is about $700 \mathrm{~m}$, sliding average thickness is about 30 $\mathrm{m}$ and volume is about 12.6 million $\mathrm{m}^{3}$.

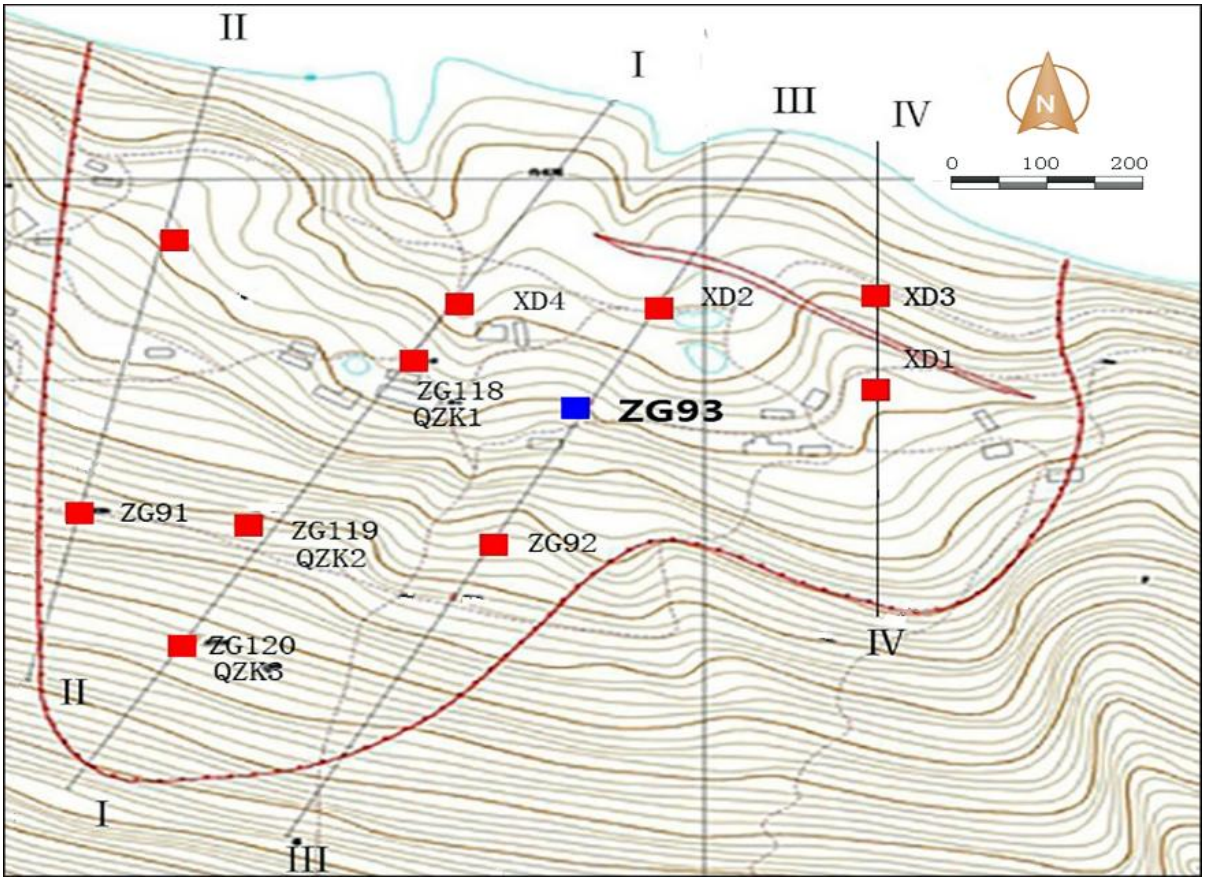

Figure 3. Baishuihe landslide monitoring sites and location map

This paper uses of Baishuihe lands lide in monitoring time period of January 2006 to December 2008, the cumulative displacement as a time series of the original sample. And from January 2006 to December 2007, the displacement as the initial sample, in January 2008 to December 2008 as a forecast of the displacement of the test sample. TABLE 1 gives the Baishuihe landslide displacement monitoring of lands lide NG93 point: 
TABLE I. NG93 MONITORING POINT OF BAISHUIHE LANDSLIDE ACCUMULATIVE DISPLACEMENT VALUE

\begin{tabular}{|c|c|c|c|c|c|}
\hline Time & $\begin{array}{c}\text { Cumulative } \\
\text { Displacement (mm) }\end{array}$ & Time & $\begin{array}{c}\text { Cumulative } \\
\text { Displacement (mm) }\end{array}$ & Time & $\begin{array}{c}\text { Cumulative } \\
\text { Displacement (mm) }\end{array}$ \\
\hline $\begin{array}{c}2006.0 \\
1\end{array}$ & 447.4 & 2007.01 & 665.3 & 2008.01 & 1557.8 \\
\hline $\begin{array}{c}2006.0 \\
2\end{array}$ & 456.2 & 2007.02 & 671.3 & 2008.02 & 1559.8 \\
\hline $\begin{array}{c}2006.0 \\
3\end{array}$ & 459.1 & 2007.03 & 686.7 & 2008.03 & 1560.8 \\
\hline $\begin{array}{c}2006.0 \\
4\end{array}$ & 485.4 & 2007.04 & 694.8 & 2008.04 & 1563.9 \\
\hline $\begin{array}{c}2006.0 \\
5\end{array}$ & 528.9 & 2007.05 & 775.9 & 2008.05 & 1589 \\
\hline $\begin{array}{c}2006.0 \\
6\end{array}$ & 550.7 & 2007.06 & 981.5 & 2008.06 & 1716.33 \\
\hline $\begin{array}{c}2006.0 \\
7\end{array}$ & 606.1 & 2007.07 & 1336.9 & 2008.07 & 1771.44 \\
\hline $\begin{array}{c}2006.0 \\
8\end{array}$ & 612.8 & 2007.08 & 1531.7 & 2008.08 & 2039.36 \\
\hline $\begin{array}{c}2006.0 \\
9\end{array}$ & 631.1 & 2007.09 & 1536.7 & 2008.09 & 2059.16 \\
\hline $\begin{array}{c}2006.1 \\
0\end{array}$ & 645.9 & 2007.10 & 1546.2 & 2008.10 & 2056.1 \\
\hline $\begin{array}{c}2006.1 \\
1\end{array}$ & 649.1 & 2007.11 & 1552.2 & 2008.11 & 2008.12 \\
\hline $\begin{array}{c}2006.1 \\
2\end{array}$ & 655.6 & 2007.12 & 1557.6 & & \\
\hline
\end{tabular}

From TABLE 1, it can be seen that the displacement data of the landslide is monotonically increasing, so the model of cusp mutation [5] is used to calculate the failure time of the prediction. Take the first 6 data to start the calculation, each additional point calculated once. The calculation shows that the correlation coefficient of the equation is above 0.99 . It is appropriate to intercept the 6 terms. In June, 2007 and July, 2007, while there has been an unstable state, indicating landslide monitoring points within ZG93 this area at this time of instability for the whole state. Then in August to December of 2007, the area of monitoring points showed a stable state, which indicates that the slope is moving to the steady state.

For the ZG93 observation point, the landslide displacement data are taken in order to carry out the mutation test, in June, 2007, the judgment value is less than 0 , the occurrence of mutation, time interval value 9.8; In July, 2007, the value of judgment is less than 0 , and the time interval is 9.38. In August, 2007, the value of judgment is less than 0 , and the time interval is 9.44;

Taking into account that the more data points to participate in the calculation, the closer landslide mutate, the more reliable the calculated interval values. So, without residual landslide amended mutate time around June 13, 2008.
TABLE II. THE VALUE OF THE RESIDUAL CORRECTION VALUE

\begin{tabular}{|c|c|c|c|}
\hline Time & $\begin{array}{c}\text { Displacement( } \\
\text { mm) }\end{array}$ & Time & $\begin{array}{c}\text { Displacement( } \\
\text { mm) }\end{array}$ \\
\hline 2006.01 & 9.942222 & 2007.01 & 14.78444 \\
\hline 2006.02 & 10.13778 & 2007.02 & 14.91778 \\
\hline 2006.03 & 10.20222 & 2007.03 & 15.26 \\
\hline 2006.04 & 10.78667 & 2007.04 & 15.44 \\
\hline 2006.05 & 11.75333 & 2007.05 & 17.24222 \\
\hline 2006.06 & 12.23778 & 2007.06 & 21.81111 \\
\hline 2006.07 & 13.46889 & 2007.07 & 29.70889 \\
\hline 2006.08 & 13.61778 & 2007.08 & 34.03778 \\
\hline 2006.09 & 14.02444 & 2007.09 & 34.14889 \\
\hline 2006.10 & 14.35333 & 2007.10 & 34.36 \\
\hline 2006.11 & 14.42444 & 2007.11 & 34.49333 \\
\hline 2006.12 & 14.56889 & 2007.12 & 34.61333 \\
\hline
\end{tabular}

For residual value, followed by taking landslide displacement data mutation testing, corresponding in June 2007, judge value is less than 0 , mutation, interval value of 0.1 ; corresponding in July of 2007, the judging value is less than 0 , mutation and time interval is 0.12 ; in August of 2007, the judging value is less than 0 , mutation and interval is 0.2 ;

After the comprehensive evaluation of the results of the residual correction, the time of the landslide is about June 20, 2008. According to the actual test results, in the night of June 19, 2008, the displacement of the mutation of the region, so the occurrence of the mutation [6] of the more accurate time prediction.

\section{CONCLUSIONS}

This method can be used in displacement observation sequence which is the certain trend of the mutation theory[7] to predict and can also be used in displacement observation sequence is random 
oscillations of the grey mutation prediction theory, giving the corres ponding slope stability criterion and the failure time and mutation displacement prediction formula. By checking Baishuihe landslide analysis shows that the prediction method is effective [8].

The characteristics of the mutation theory [9] forecast method is: (1) Landslide is obviously a non-continuous mutation phenomenon, with the mutation theory to describe the landslide phenomena reasonable than describe the phenomenon of continuous mathematical tools for any kind of existing; (2) It reflects the dynamic tracing and forecasting thoughts[10]; (3) It has a dual function, can do the stability analysis, and forecast the time and displacement; (4) It can reproduce the evolution of slope; (5) Time prediction may send a few months in advance.

\section{ACKNOWLEDGEMENTS}

This research is funded by the Comprehensive Control Technology in Jiaozuo City, resource-exhausted cities Geological Environment (No.2014056061) and National Natural Sciences Foundation of China (No. 41302278). The authors wishes to thank the teachers for funding his research.

\section{REFERENCES}

[1] J. Qin Siqing.Nonlinear catastrophic model of slope instability and chaotic dynamics mechanism of slope evolution process.
Chinese Journal of Rock Mechanics and Engineering, 2000, 19(4): $486-492$.

[2] J. Xu Zenghe, Xu Xiaohe. Instability of fault earthquake in rheological media and cusp catastrophe. Rock and Soil Mechanics, 2000, 21(1): 24-27.

[3] J. Cheng Xue-jun,Cai Mei-feng.Li Chang-hong.gray-catast tophic model and its application in axoustic emission monit oring[J].China Mining, 1997,(3):37-39.)

[4] M. Jiang Deyi Zhu Hehua Du Yungui Stability anaiysis and prevention study on landside.Chongqing Chongqing University Press 2005:152-154)

[5] J. AN Yu-hua, WANG Qing, ZHANG Chen, et al. Risk degree evaluation of debris flows based on cat astrophic theory. Journal of Jilin University (Earth Science Edition), 2012, 42(Supp.1): $355-361)$

[6] J. Xiong Chuanxiang, Gong Xiaonan, Wang Chenghua. A Deformation energy catastrophic model of high-speed landslide before sliding .Journal of Zhejiang University (Engineering Science), 2000, 34(4): 443-447.

[7] J . WANG Cheng-hua, KONG Ji-ming. The differentiating danger slope occurring high-speed landslide. Journal of Engineering Geology, 2001, 9(2): 127-132.

[8] J. WANG Shuren, ZHANG Yanbo. New method for landslide forecast and its application based on longitudinal data and catastrophic theory [. Journal of China Coal Society, 2009, 34(5) : 640-644.

[9] J. Yin Youquan, Du Jing. Coattail catastrophe model in the process of earthquake. Acta Seismologica Sinica, 1994, 16(4): $416-422$.

[10] M. Tang Chun an,Catastrophe in rock unstable failure. BeiJing:China Coa In Industry Publishing House, 1993: 112-121. 